\title{
On the Bulk Modulus of Open Cell Foams
}

\author{
B. Moore, T. Jaglinski, D.S. Stone ${ }^{\S}$ and R.S. Lakes* \\ *Department of Engineering Physics and \$Department of Materials Science, University of \\ Wisconsin-Madison, 541 Engineering Research Building \\ 1500 Engineering Drive, Madison, WI 53706-1687 USA
}

Received: 29 January 2007 Accepted: 26 February 2007

\begin{abstract}
Bulk properties of open cell polyurethane foam are studied in a hydrostatic compression experiment under strain control. A linear region of behaviour is observed in the stress-strain curve, followed by a non-monotonic region corresponding to a negative incremental bulk modulus. The bulk modulus in the linear region is in reasonable agreement with the value calculated from compressional Young's modulus and Poisson's ratio. The linear region of behaviour in hydrostatic compression corresponds to less than half the axial strain range observed in axial compression.
\end{abstract}

\section{INTRODUCTION}

Most experimental studies of foam properties have been conducted in tension/compression ${ }^{(1)}$. In compression of a flexible polymer foam, a linear region in the load-deformation curve is observed up to about $5 \%$ strain. This region is associated with bending ${ }^{(2)}$ of the cell ribs, though early models advocated rib extension $^{(3)}$. Rib bending gives rise to a modulus which increases as the square of the relative density in the linear region ${ }^{(4)}$. If the foam does not deviate too much from isotropy, the Poisson's ratio is close to 0.3. At higher compressive strain, there is a plateau region of reduced slope in the stress-strain curve due to buckling of the cell ribs, followed by densification in which the curve's slope increases due to contact of the cell ribs. In the plateau region, localization of strain has been observed ${ }^{(5)}$ and interpreted in the context of stability ${ }^{(6,7)}$. As for tension, a linear region in the load-deformation curve is observed, followed by a region of increasing slope due to cell rib orientation.

*To whom correspondence should be addressed

${ }^{\circ}$ Rapra Technology, 2007 
Hydrostatic compression of foam is of interest in a variety of contexts including under-sea applications. Syntactic foam, for instance, consists of hollow glass inclusions in an epoxy matrix ${ }^{(8,9)}$. Such foam is intended to have a bulk modulus comparable to that of water (about $2 \mathrm{GPa}$ ), and sufficient compressive strength. This foam is not permeable to water since the cells do not have communicating paths. A syntactic foam was found to have ${ }^{(10)}$ a Young's modulus of $\mathrm{E}=$ 2.76 GPa and a shear modulus of $\mathrm{G}=1.03 \mathrm{GPa}$, The Poisson's ratio $v$ based on the isotropic relation

$$
\mathrm{E}=2 \mathrm{G}(1+v)
$$

was $v=0.33$, typical of a normal solid.

Indentation of foam, in contrast to homogeneous material, depends upon the compressibility ${ }^{(11,12)}$. Strength measurement in triaxial compression, including hydrostatic compression is relevant to understanding how foam responds to impacts $^{(13)}$, particularly in the application of closed cell foam in packaging. The bulk modulus of these foams is less than the prediction of theoretical models, as a result of influence by wrinkles in the cell walls ${ }^{(14)}$. Strength measurements ${ }^{(15)}$ in hydrostatic compression have also been done on closed cell polymeric structural foam (Rohacell) in the evaluation of its crushing behavior. Related studies of strength have been conducted of multiaxial yield of aluminum alloy foam ${ }^{(16)}$.

In contrast to syntactic foam and other closed cell foams, open cell foams are permeable to fluids; but the properties of such foams under hydrostatic stress are of interest for other reasons. Triaxial compression (of equal magnitude in three directions) of open-cell foam is done in the preparation of negative Poisson's ratio foams ${ }^{(17)}$, however little is known about the behaviour of open cell foams under triaxial compression. Triaxial compression of foam is also of interest since such stress states can occur in applications of foam such as compression under a transverse constraint as well as in localized indentation of foam. Open-cell foams have been studied analytically to determine their moduli. It has long been known that the Young's modulus is proportional to the square of the relative density, i.e. the foam density divided by the density of its ribs. Careful analysis ${ }^{(18)}$ of foam cells as tetrakaidecahedra (polyhedra of 14 faces) provides the constant of proportionality. This lattice analysis is elastically isotropic; it also predicts the bulk modulus to vary linearly with the relative density, so for low density foam, the bulk modulus greatly exceeds the Young's modulus or the shear modulus, and the Poisson's ratio is predicted to approach 0.5 as the density becomes small. 
Inference of the bulk modulus from Young's modulus and Poisson's ratio from tensile tests depends upon isotropy of the material. Similarly, inference of the bulk modulus from Young's modulus and shear modulus depends on isotropy. Foams generally deviate to a lesser or greater extent from isotropy. It is not obvious how much deviation from isotropy is tolerable for such inference. Even when anisotropy of foam is explored, the full set of elastic constants which would be needed for such judgment, is not captured. Therefore it is preferable to directly measure the bulk modulus.

In the present study the properties of open-cell foam under uniaxial compression and hydrostatic compression are studied, and bulk modulus is determined. Experiments are performed under displacement (uniaxial or volumetric) control.

\section{MATERIALS AND METHODS}

Flexible polymer foams were obtained from Foamade Industries, (Auburn Hills, $\mathrm{MI}$ ), and from Foamex International Inc (Eddystone, PA). These foams were of a low density $\left(0.03 \mathrm{~g} / \mathrm{cm}^{3}\right)$ open-cell reticulated structure with pore sizes of $2.5 \mathrm{~mm}$ (10 pores per inch (ppi)) and $0.4 \mathrm{~mm}$ (60 ppi). Cubes, cylinders and spheres were cut from the bulk foam via an iterative sectioning process. Specifically, portions of foam were sectioned and the specimen measured. Irregularities were further sectioned. Specimens included cubes with side lengths of about $2.5 \mathrm{~cm}$, cylinders were about $3 \mathrm{~cm}$ in diameter and $4.5 \mathrm{~cm}$ long, and spheres were about $3.5 \mathrm{~cm}$ in diameter.

Uniaxial compression studies were conducted using a MTS (Minneapolis, MN) screw driven test machine with a $45 \mathrm{~N}(10 \mathrm{lb})$ load cell. Engineering strains were calculated from cross head displacement and specimen length. Young's modulus was determined from the initial slope of the load-deformation curve. Tests were conducted in different directions at a slow rate $(0.5 \mathrm{in} / \mathrm{min})$ to determine the degree of anisotropy. Uniaxial deformation curves were compared with those due to volumetric deformation.

Hydrostatic compression studies were performed by applying water pressure via a plunger device. Since no standard methods are known for hydrostatic compression of open cell foam, the test method was developed for this study. The foam specimen was placed in a chamber (1 liter, polycarbonate bottle (Nalgene, Rochester, NY)) which was then filled with water. Ingress of water into the foam was prevented by enclosing the foam in a rubber membrane. Initially, cubic specimens were enclosed in flat latex membranes $0.15 \mathrm{~mm}$ thick 
(McMaster-Carr, Chicago, IL) cemented together in segments. This approach, however, resulted in frequent leaks of water. Therefore, spherical or cylindrical foam specimens were enclosed in cylindrical rubber membranes $0.076 \mathrm{~mm}$ thick (Trojan, Church \& Dwight Co., Princeton, NJ). The specimen was placed at the end of the membrane, then a $3 \mathrm{~mm}$ diameter air relief tube was placed into the membrane (Figure 1). The membrane was twisted around the tube to take up the slack and to remove as much air as possible. Effort was made to minimize pre-compression of the specimen. Several windings of electrical tape were tightly wrapped around the tube and membrane to seal it, then the excess latex was trimmed and removed. The pressure chamber was prepared as follows. The screw top was modified by drilling a hole through the cap and adding a bulkhead joint to provide a water injection port. An air relief tube was provided to allow the air within the foam to escape as it was compressed. Known volumes of water were injected using a calibrated screw piston. Gauge pressure was measured using a digital HHP-2021 manometer (Omega) with a $13 \mathrm{kPa}$ operating range. The manometer uses an input tube of $4 \mathrm{~mm}$ inner diameter and $1 \mathrm{~mm}$ wall thickness. The manometer is only capable of reading air pressure so the last section of the manometer tube, roughly $10 \mathrm{~cm}$, was filled with air rather than water. Water has a much higher bulk modulus (about $2 \mathrm{GPa})$ than the foam $(\sim 50 \mathrm{kPa})$; moreover the structural stiffness of the bottle greatly exceeds that of the foam. Therefore this experimental setup is in effect a nearly perfect, displacement controlled test machine.

To conduct a hydrostatic compression test, the chamber was filled with tap water and the lid was placed tightly onto the chamber. The chamber was then topped

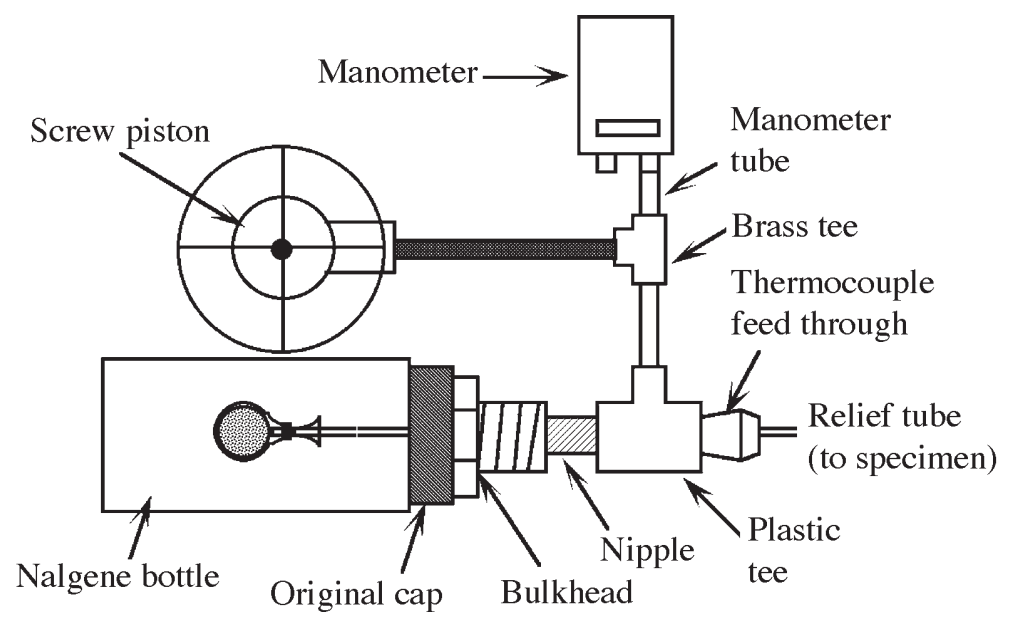

Figure 1. Experimental apparatus 
off with water. When the water level reached the thermocouple feedthrough hole, the fitting was replaced and the chamber was turned upside down and agitated to release any remaining air bubbles. The chamber was then placed upright, the thermocouple feed through removed, and water added as needed. This process was repeated until all the visible air bubbles were removed from the system. Once the system was free of bubbles the manometer was attached and the initial pressure reading recorded. The volume control screw was backed off to inflate the membrane beyond the diameter of the sample. Water was then added ("displaced") into the system in increments of about $0.14 \mathrm{~mL}$. After each displacement increment the manometer was allowed to settle to a constant pressure. A compression test to $40 \%$ strain typically took up to 5 hours. A zero strain point was inferred based on the slope change during the inflation of the latex rubber. The initial pressure was sufficiently small that the only effect seen was a seating effect, associated with a toe region at small pressure in the graphs.

After testing, each specimen was removed from the pressure chamber, unwrapped and allowed to recover for at least ten times the duration of the test. For consecutive tests the specimen was wrapped in a new membrane for each test.

\section{RESULTS AND DISCUSSION}

\section{Uniaxial Compression}

Results of uniaxial compression experiments on small-cell $(0.4 \mathrm{~mm})$ foam are shown in Figure 2. As is typical of low-density polymer foams, a linear elastic region up to $5 \%$ to $10 \%$ strain was observed following an initial seating regime of reduced slope. The seating region of the curve, concave up, is attributed to the irregularity of the foam surface. These irregularities are flattened out before the foam as a whole is significantly compressed. The foams exhibited a plateau and a region of increased slope at progressively higher strain. Young's moduli of the $25.4 \mathrm{~mm}, 60$ ppi cube (Figure 2) were 47, 34 and $37 \mathrm{kPa}$ for the three orthogonal directions. The anisotropy ratio was modest: 1.0:1.1:1.4. Since these specimens were cubical rather than slender, inference of Young's modulus involves a correction for the constraint at the ends. Assuming a rough contact and a Poisson's ratio of 0.3 , the correction ${ }^{(19)}$ is less than $3 \%$.

The larger cell foams ( $2.5 \mathrm{~mm}$ cell, $10 \mathrm{ppi})$ showed a more pronounced directional dependence than the small cell $(0.4 \mathrm{~mm})$ foam. Young's moduli were 9,9 and $20 \mathrm{kPa}$ in a cubical $25.4 \mathrm{~mm}$ sample, or ratios of 1.0:1.0:2.2. A $50.8 \mathrm{~mm}$ cube of the same foam had Young's moduli of 15,15 , and $37.5 \mathrm{kPa}$, or ratios of 1.0:1.0:2.5. The anisotropy is due to the elongated pore shape in the foams of 


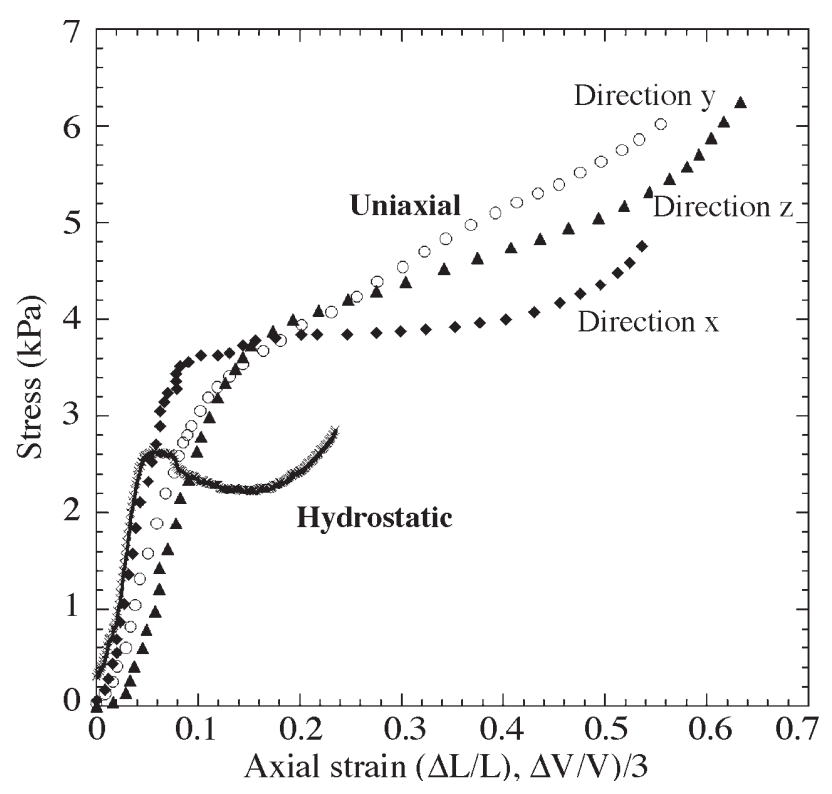

Figure 2. Response of $0.4 \mathrm{~mm}$ cell-size foam to uniaxial compression and to hydrostatic compression

larger cell size. A small specimen of large cell foam has many incomplete cells at the surfaces, hence a lower apparent modulus. The effect of incomplete cells is opposite to the stiffening effect observed in slender specimens ${ }^{(10)}$ in bending or torsion. The latter effect results from distributed moments in the cell ribs; it has been understood in the context of generalized continuum mechanics. Typical stress-strain curves are shown in Figure 3. The anisotropy is manifested in the shape of the plateau region as well as the slope in the linear elastic region. Observe that there is no "yield" when compressing normal to the elongated direction. In the elongated direction there is a slight non-monotonic behaviour. The test is evidently sufficiently sensitive to detect the buckling of single cells or groups of cells. The cell size in this case is one tenth the small specimen cube length. The structure of the foams is shown in Figure 4. Figure 4 (c) shows the elongation of the cells associated with anisotropy.

\section{Hydrostatic Compression}

Hydrostatic compression of small cell (60 ppi) foam resulted in a pressurevolume characteristic as shown with the uniaxial compression results in Figure 2. The slope in the linear, post-seating region corresponds to a bulk modulus of $26 \mathrm{kPa}$. At large strain, beyond the linear region, the behaviour 


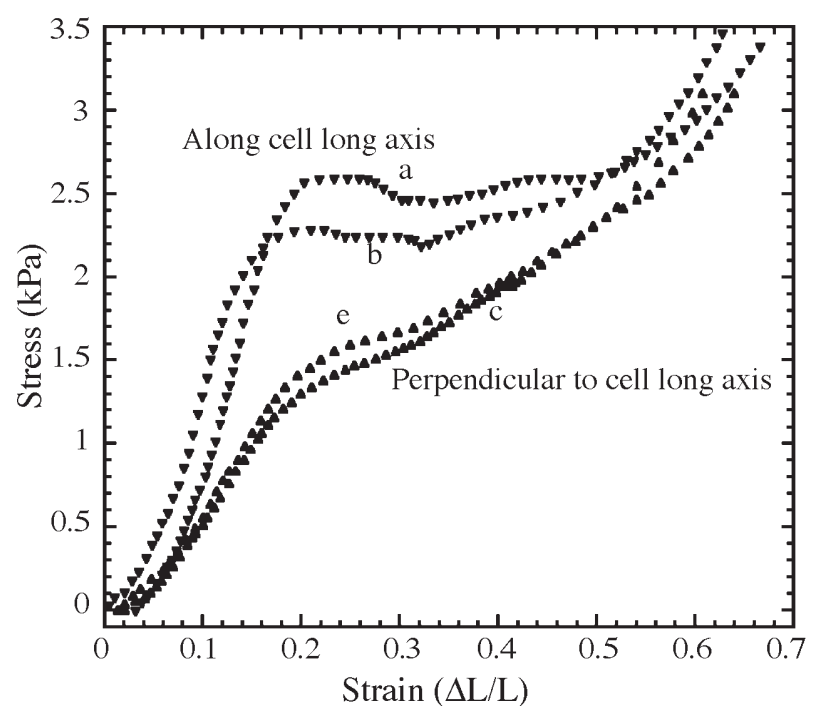

Figure 3. Response of $25 \mathrm{~mm}$ cube of $2.5 \mathrm{~mm}$ cell-size foam to uniaxial compression. Several tests are indicated by letters, a, b, c, e
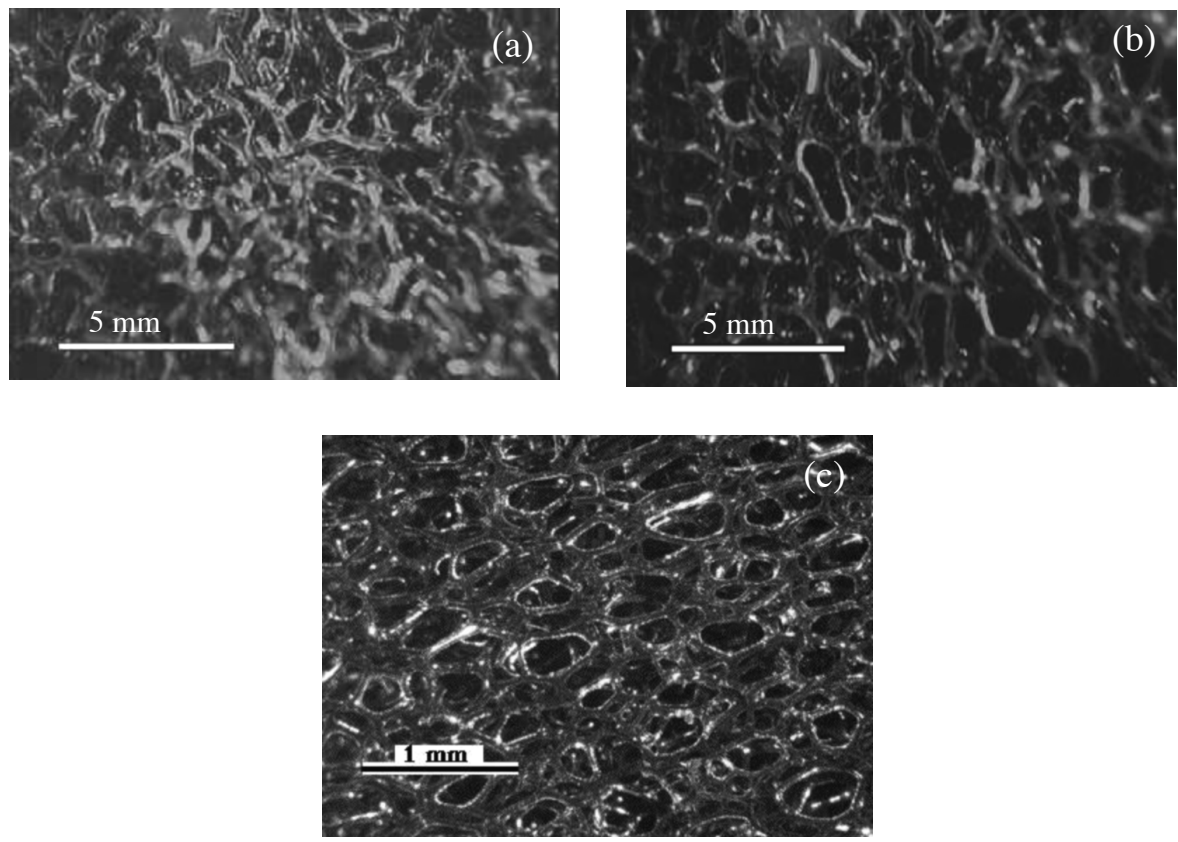

Figure 4. Structure of collapsed foams. (a) Negative Poisson's ratio foam produced by symmetric triaxial compression, scale bar, $5 \mathrm{~mm}$; (b) Conventional foam under uniaxial compression, scale bar, 5 mm; (c) Anisotropic foam, scale bar, 1 mm 
is non-monotonic in contrast to the uniaxial case. The negative slope in this region corresponds to a negative incremental bulk modulus of about - $9 \mathrm{kPa}$. Further details and a discussion of the implications are presented elsewhere ${ }^{(20)}$. Briefly, we recognize that while a negative modulus entails instability, it can be stabilised by a surface constraint as achieved in the experiments.

The bulk modulus $\mathrm{K}$ is related to Young's modulus $\mathrm{E}$ in isotropic materials by;

$$
\mathrm{K}=\mathrm{E} / 3(1-2 v)
$$

with $v$ as Poisson's ratio. For a typical foam Poisson's ratio of $0.3, \mathrm{~K}=0.83 \mathrm{E}$. If Poisson's ratio is 0.33 , then $\mathrm{K}=0.98 \mathrm{E}$. A direct comparison between axial and hydrostatic results is complicated by the fact that even the small cell foam is not fully isotropic. Even so, $\mathrm{E}=34 \mathrm{kPa}$ in the most compliant direction for small cell (60 ppi) foam corresponds (with a Poisson's ratio 0.3 ) to $\mathrm{K}=27 \mathrm{kPa}$ compared with the observed $26 \mathrm{kPa}$. This is a reasonable agreement in view of the slight anisotropy. The direct measurement of bulk modulus performed here confirms the impression of a moderate value of bulk modulus based on the known Poisson's ratio of foam. The theoretical prediction ${ }^{(6)}$ of large bulk modulus is not confirmed. The theory, though mathematically elegant, makes the simplifying assumption that the foam ribs are straight. Therefore the ribs in the model foam deform axially when the foam is subject to hydrostatic stress hence a prediction of bulk modulus linear in the relative density. The ribs deform in bending if the foam is sheared or deformed axially, hence a prediction of $\mathrm{G}$ and $E$ quadratic in the relative density. In reality, the foam ribs are curved, so they deform in bending in all modes of macroscopic deformation.

The maximum stress corresponding to elastic collapse in elastomeric foam in hydrostatic compression is predicted to be 0.83 of the value for uniaxial compression $^{(21)}$. In comparison, the ratio in the experiment is about 0.77 . The difference may be attributed to the slight anisotropy of the small cell foam or to its rate dependence.

\section{Nonlinearity and Buckling}

As for ranges of linearity and nonlinearity, we recall that the volumetric strain is the sum of the axial strains $\Delta \mathrm{L} / \mathrm{L}$. For an isotropic material, the axial strain is therefore one third the volumetric strain. The linear region for hydrostatic compression corresponds to about $7 \%$ volumetric strain. One third of this is the calculated axial strain, $2.3 \%$. This is substantially less than the axial strain range of more than $5 \%$ observed in the linear region of the uniaxial compression curves. This discrepancy is explained in the context of the buckling of cell 
ribs. In uniaxial compression, the cells can bulge laterally, manifesting the Poisson effect before rib buckling occurs. In hydrostatic compression, this bulge cannot occur. Therefore buckling occurs more abruptly. It is likely that the ribs experience a higher local strain in this sort of buckling than in uniaxial compression. Such a notion is consistent with the large hysteresis and cycle dependence seen in hydrostatic compression ${ }^{(20)}$. Volumetric strain used in the manufacture of negative Poisson's ratio foam was a factor of 3 to a factor of 5. This is beyond the linear range of stress-strain behaviour and is also beyond the non-monotonic range of hydrostatic pressure-volume behaviour. Permanent volumetric compression in making negative Poisson's ratio foam gives rise to a post-buckled cell structure with no preferred orientation (Figure 4a) in contrast to the oriented cell structure, which occurs in uniaxial compression (Figure $4 \mathrm{~b}$ ) by a factor of two in the left-right direction. The structural difference is associated with the difference in behaviour of the foam in hydrostatic compression in comparison with uniaxial compression.

\section{CONCLUSIONS}

Hydrostatic compression of open cell foam discloses a linear range of behaviour. The bulk modulus is in reasonable agreement with the value calculated from Young's modulus and Poisson's ratio assuming isotropy. The linear region for hydrostatic compression corresponds to about $7 \%$ volumetric strain, or $2.3 \%$ axial strain, which is in contrast to uniaxial compression for which the linear range corresponds to $5 \%$ or more axial strain. Beyond about $20 \%$ volumetric strain in hydrostatic compression, the pressure-volume curve has a nonmonotonic region indicative of a negative incremental bulk modulus.

\section{REFERENCES}

1. Gibson, L.J. and Ashby, M.F., Cellular Solids: Structure and Properties, Cambridge: Cambridge University Press (1997).

2. Ko, W.L., Deformations of foamed elastomers, J. Cell. Plast., 1, (1965) 45-49.

3. Gent, A.N. and Thomas, A.G. The deformation of foamed elastic materials, $J$. Appl. Polym. Sci., 1, (1959) 107-112.

4. Gibson, L.J. and Ashby, M.F., The mechanics of three-dimensional cellular materials, Proc. R. Soc. Lond. A, 382, (1982) 43-56.

5. Stronge, W.J. and Shim, V.P.W., Microdynamics of crushing in cellular solids, J. Engineering Materials Technology, 110, (1988) 185-190. 
6. Lakes, R.S., Rosakis, P. and Ruina, A., Microbuckling instability in elastomeric cellular solids, J. Materials Science, 28, (1993) 4667-4672.

7. Abeyaratne, R. and Triantafyllidis, N. An investigation of localization in a porous elastic material using homogenization theory, J. Applied Mechanics, 51, (1984) 481-486.

8. Resnick, I., A buoyancy material for undersea depths. U. S. Patent 3,477,967, (1969).

9. Lee, K.J. and Westmann, R.A., Elastic properties of hollow-sphere-reinforced composites, Journal of Composite Materials 4, (1970) 242-253.

10. Lakes, R.S., Experimental microelasticity of two porous solids, International Journal of Solids and Structures, 22, (1986) 55-63.

11. Shaw, M.C. and Sata, T., The plastic behavior of cellular materials, Int. J. Mech. Sci., 8, (1966) 469-478.

12. Wilsea, M., Johnson, K.L. and Ashby, M.F., Indentation of foamed plastics, Int. J. Mech. Sci., 17, (1975) 457-460.

13. Maji, A.K., Schreyer, H.L., Donald, S., Zuo, Q. and Satpathi, D., Mechanical properties of polyurethance foam impact limiters, Journal of Engineering Mechanics, 121, (1995) 528-540.

14. Masso Moreu, Y. and Mills, N.J., Rapid hydrostatic compression of low density polymeric foams, Polymer Testing, 23, (2004) 313-322.

15. Li, Q.M., Mines, R.A.W. and Birch, R.S., The crush behavior of Rochacell-51WF structural foam, International Journal of Solids and Structures, 37, (2000) 63216341.

16. Sridhar, I. and Fleck, N.A., The multiaxial yield behaviour of an aluminum alloy foam, Journal of Materials Science, 40, (2005) 4005-4008.

17. Lakes, R.S., Foam structures with a negative Poisson's ratio, Science, 235, (1987) 1038-1040.

18. Zhu, H.X., Knott, J.F. and Mills, N.J., Analysis of the elastic properties of open-cell foams with tetrakaidecahedral cells, J. Mech. Phys. Solids, 45, (1997) 319-343.

19. Chau, K.T., Young's modulus interpreted from compression tests with end friction, J. Engineering Mechanics (ASCE), 123, (1997) 1-7.

20. Moore, B., Jaglinski, T., Stone, D.S. and Lakes, R.S., Negative incremental bulk modulus in foams, Philosophical Magazine Letters, 86, (2006) 651-659.

21. Triantafilou, T.C., Zhang, J., Shercliff, T.L., Gibson, L.J. and Ashby, M.F., Failure surfaces for cellular materials under multiaxial loads II. Comparison of models with experiment, Int. J. Mechanical Sci., 31, (1989) 665-678. 